\title{
Micro-strip metal detector for the beam profile monitoring
}

\author{
V. Pugatch ${ }^{\mathrm{a}}$, M. Borysova ${ }^{\mathrm{a}}$, A. Mykhailenko ${ }^{\mathrm{a}, *}$, O. Fedorovitch $^{\mathrm{a}}$, Y. Pylypchenko ${ }^{\mathrm{a}}$,

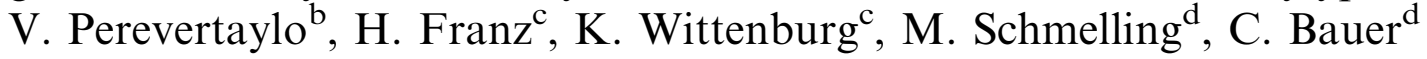 \\ ${ }^{a}$ Kyiv Institute for Nuclear Research, Kyiv, Ukraine \\ binstitute of Micro Devices, Kyiv, Ukraine \\ ${ }^{\mathrm{c}}$ Deutsches Elektronen-Synchrotron, Hamburg, Germany \\ ${ }^{\mathrm{d}}$ Max-Planck-Institute for Nuclear Physics, Heidelberg, Germany
}

Available online 7 August 2007

\begin{abstract}
The Micro-strip Metal Detector (MMD) design and production technology, readout electronics as well as areas of applications are described. The MMD was designed for beam profile monitoring of charged particle and synchrotron radiation beams. Using photolithography and plasma-chemistry etching technologies we succeeded in creating detectors with a metal strip's thickness of less than $2 \mu \mathrm{m}$ and without any other materials in the working area. The principle of operation is based on the Secondary Electron Emission (SEE). The results obtained with the MMD at the monochromatic synchrotron radiation beam at HASYLAB (DESY) are also presented. The current version of the MMD allows measuring a beam profile and position with an accuracy of $20 \mu \mathrm{m}$.
\end{abstract}

(C) 2007 Elsevier B.V. All rights reserved.

PACS: 29.40.Wk; 07.77.Ka; 07.85.Fv

Keywords: Monitoring; Electron; Ion; Synchrotron; Profile

\section{Introduction}

At many applications of synchrotron radiation and charged particle beams there is a necessity to provide online beam profile monitoring. Among the general requirements to the Beam Profile Monitor (BPM) are high resolution, high radiation tolerance and the ability of non-destructive monitoring. For low intensity beams a silicon micro-strip detector could provide a position resolution even at the sub-micron level. However, radiation hardness aspects make this approach rather limited. The Micro-strip Metal Detector (MMD) has an advantage here because of using metal as detecting material instead of a semiconductor. The position resolution of a few micrometers is in the reach for the current manufacturing technology of the MMD and applying latest achievements in lithography a position resolution at the sub-micron level

\footnotetext{
${ }^{*}$ Corresponding author.

E-mail address: mykhailenko@kinr.kiev.ua (A. Mykhailenko).
}

could also be attained. In this paper we present results of measuring the profile of a synchrotron radiation beam at the HASYLAB (DESY) by means of the MMD.

\section{MMD principle of operation}

Passing through the metal strips, a beam of charged particles or synchrotron radiation initiates secondary electron emission (SEE) (Fig. 1). A positive charge appearing at the electrically isolated strip is integrated by a sensitive Charge Integrator (CI) [1]. To improve the extraction of secondary electrons (SE) an accelerating electric field $\vec{E}$ is created around the strip. A majority of the secondary electrons has an energy in the range of a few $\mathrm{eV}$ allowing to operate the MMD with a voltage of only $20 \mathrm{~V}$. Secondary electrons are emitted mainly from a thin $(10-50 \mathrm{~nm})$ surface layers [2,3]. Thus, one may consider the possibility to create submicron thick detectors without a significant loss of their sensitivity. 


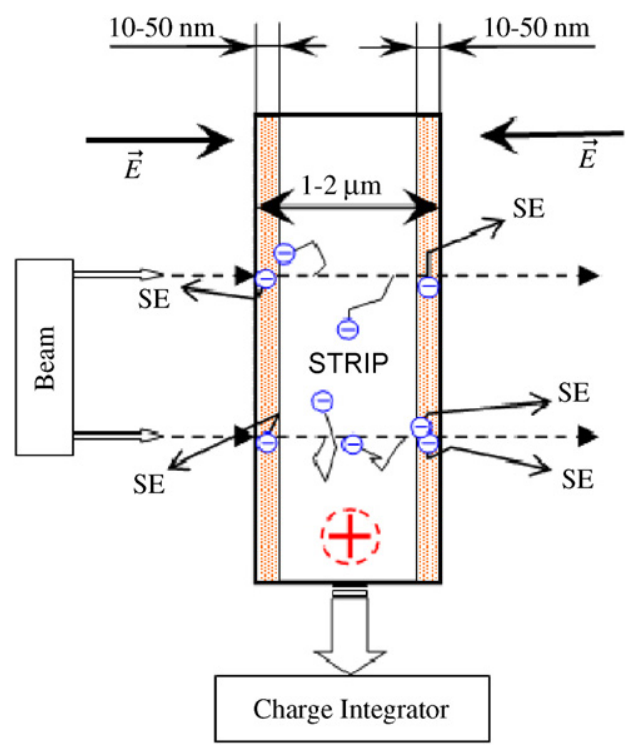

Fig. 1. MMD principle of operation.

\section{MMD production technology and technical details}

Design, technology and structure of the MMD are determined by the principle of operation and the application requirements. The main MMD feature, determining the complexity of the manufacturing process, are the micrometer sizes of the strips stretched over the working area. The strip's manufacturing, by applying plasmachemistry technologies for etching of multilayer structures, is described in detail in Ref. [4]. The MMD production technology includes micro-photolithography, plasmachemistry etching and assembling. These steps are briefly described below.

The sensor layer of the MMD prototype has 32 nickel strips $(1-2 \mu \mathrm{m}$ thick, $70 \mu \mathrm{m}$ pitch) placed on a $500 \mu \mathrm{m}$ thick silicon wafer. Every strip is connected at one end to its individual charge integrator input channel and at the other end to a stable current source of 25 or $250 \mathrm{pA}$ (baseline). This allows for checking the integrity of the strips as well as making corrections for a possible baseline variation.

\subsection{Photolithography}

On the silicon wafer ( $500 \mu \mathrm{m}$ thick) two dielectric layers are created to isolate the future metal strips from the semiconductor. At first, the silicon oxide $\left(\mathrm{SiO}_{2}, 0.1-0.3 \mu \mathrm{m}\right.$ thick) is grown and covered later by a $0.2 \mu \mathrm{m}$ thick silicon nitride $\left(\mathrm{Si}_{3} \mathrm{~N}_{4}\right)$. To improve adhesion the dielectric layer is covered with a thin titanium film (about $50 \mathrm{~nm}$ ). Finally, a $1-2 \mu \mathrm{m}$ thick nickel-film is deposited. Such a "sandwich" structure is created on both sides of the silicon wafer. On the front side, 32 strips $(40 \mu \mathrm{m}$ width, $70 \mu \mathrm{m}$ pitch) with connection pads are formed by photolithography methods, while at the back side of the Si-wafer a nickel mask is kept for the forthcoming plasma-chemistry etching.

\subsection{Plasma-chemistry etching}

To remove the silicon wafer material from the working area of the MMD (making the metal strips hanging there free) a plasma-chemical reactor at KINR with variable ion energy has been used. At first, through an additional metal foil mask, a thin surface layer $(20-80 \mu \mathrm{m})$ of silicon is removed on the front side of the wafer. Then, through the rectangular shaped window on the back side, the plasmachemical etching is performed. The initial etching speed is in the range of $2.5 \mu \mathrm{m} / \mathrm{min}$ at an ion energy of $80 \mathrm{eV}$ and a discharge current of $10 \mathrm{~A}$. When the silicon wafer thickness approaches $50-100 \mu \mathrm{m}$ the etching speed is slowed down to $0.3 \mu \mathrm{m} / \mathrm{min}$, by decreasing the current to $4 \mathrm{~A}$ and the ion energy to $20 \mathrm{eV}$. In this way, it is possible to produce the MMD with a completely removed Si-wafer in the working area $\left(10 \times 15\right.$ or $\left.6 \times 15 \mathrm{~mm}^{2}\right)$ and all 32 strips untouched. Similarly, the accelerating layers are produced. The difference is just in the number of the strips: the accelerating layers have two strips, placed at a distance which is a bit wider than the one between the outermost strips of the sensor layer.

\subsection{MMD assembling}

The sensor and the accelerating layers are each glued to the specially designed ceramics pitch adapters. These adapters are glued together. Every strip pad of the layers is connected by supersonic welding to the metallization on the ceramics, using a wire of $25 \mu \mathrm{m}$ diameter, and further through a flexible polyimide cable to the 50-pin D-Subminiature connector. The whole construction is housed in a protective metal case (see photo Fig. 2). The connection to the charge integrators is provided by $5 \mathrm{~m}$ long coaxial cables. The frequency outputs of the charge integrators, proportional to the measured beam intensity, are read out by VME-based scalers, while a corresponding software presents the data on-line on the computer display.

\section{Applications}

Nowadays micro-beams of charged particles as well as synchrotron radiation are widely used for science and technology. In many of these applications there is a necessity to know the distribution of the intensity in the beam. In comparison with other detectors, the MMD has advantages like:

- Low thickness of the detecting material (less than $1-2 \mu \mathrm{m})$.

- High sensitivity and minimal disturbance to the beam,

- Low operating voltage $(20 \mathrm{~V})$.

- Simple data acquisition (charge integrators, scalers, computer).

- High radiation tolerance (gigarad level).

- High position resolution $(20 \mu \mathrm{m}$ now and down to $1 \mu \mathrm{m}$ in the near future). 
In view of these advantages one may consider the MMD as a perspective device for the profiling of micro-beams. We consider that the MMD could be also used for intensive beams of high energy charged particles, as a feedback element for stabilizing and/or focusing collider beams. Indeed, even if the central part of the detector is burnt out the remaining strips operating in the beam halo would provide necessary feedback signals. We have also

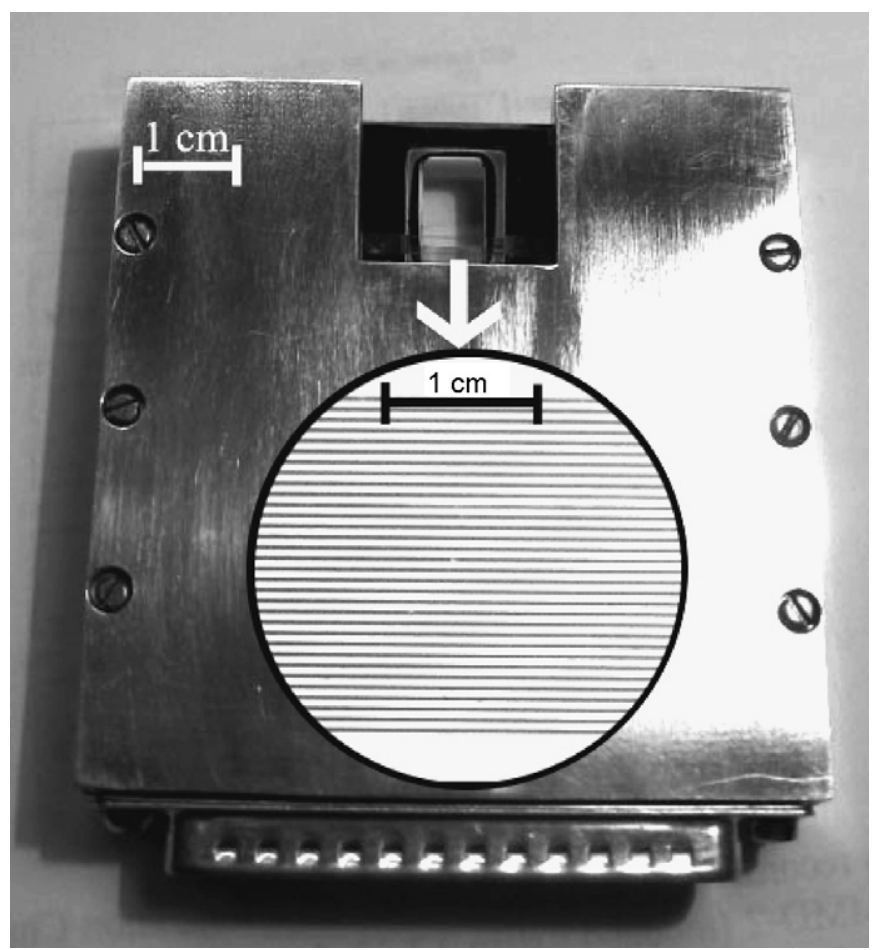

Fig. 2. MMD view. There are enlarged sensor strips in the circle (32 strips: $40 \mu \mathrm{m}$ width, $70 \mu \mathrm{m}$ pitch). performed studies demonstrating a good performance of the MMD in the focal plane of a mass-spectrometer.

\section{Experimental results}

During the development of the MMD production technology we carried out several experiments with different prototypes of the detector. First the MMD was used for the on-line control, positioning and focusing of a $32 \mathrm{MeV}$ alpha-particle beam at the Tandem generator used for studies of the single events upsets of the BEETLE chip (MPIfK, Heidelberg) [5]. Other measurements were performed with a monochrome synchrotron radiation beam of the Low Energy Beamline PETRA 1 HASYLAB (DESY). The beam has a mean energy of about $20 \mathrm{keV}$ and intensities of up to $10^{12} \mathrm{ph} /\left(\mathrm{s} \times \mathrm{mm}^{2}\right)$. Previous results obtained at HASYLAB are described in Ref. [6].

We have been exploiting two styles of measurements: in dynamic mode, where a change of beam position or beam size was simulated, and in static mode, respectively.

\subsection{Measurements of X-ray beam profile in the atmosphere}

The monochrome synchrotron beam traversed a special beryllium window at the end of the vacuum pipe and continued in the atmosphere. At a distance of $20 \mathrm{~cm}$ from the beryllium window, the beam passed through vertical and horizontal slits, the MMD and an Ionization Chamber (IC) were located downstream. We were enabled to change the slits simulating changes of the beam size. By means of the IC we could determine the intensity of the passing beam.

The results had showed that the MMD was able to measure the beam profile, but there were signal distortions because of air ionization. Opening the slit step-by-step, we

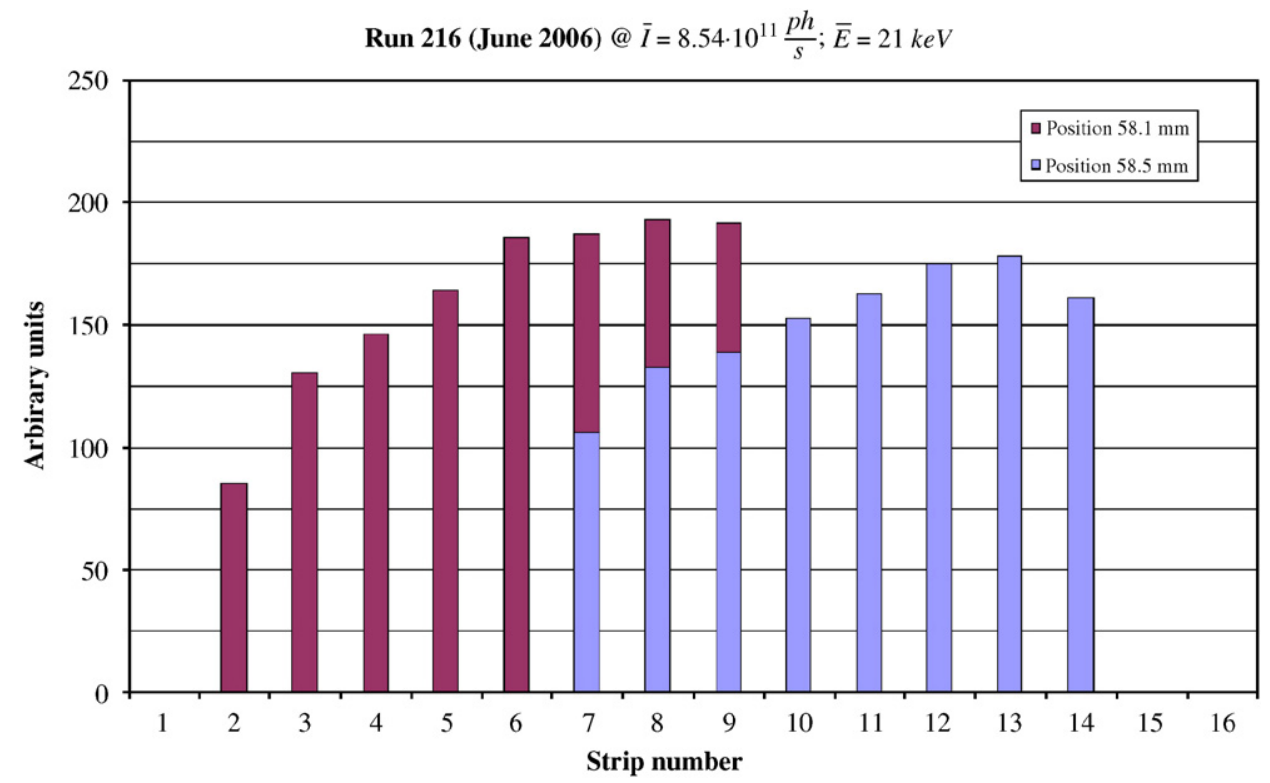

Fig. 3. Two beam profiles measured at different positions of the MMD in dynamic mode. The distance between the two detector positions is $400 \mu \mathrm{m}$. 
observed an increasing of the signals at all strips simultaneously, in spite of the fact that the beam was hitting just a few strips. Also, due to air ionization, the calculated conversion coefficient (i.e. the ratio of incident photons to secondary electrons) was found to be equal to a few photons per one electron, whereas the order of magnitude for this coefficient in vacuum is about $5000 \mathrm{ph} / \mathrm{e}^{-}$[6]. Summarizing, we can conclude that we did not obtain accurate information about the beam profile in the atmosphere, but it was still possible to work with the low intensity beams, determining their intensity and core position.

\subsection{Measurements of X-ray beam profile in vacuum}

In this experiment the MMD was placed inside the vacuum chamber standing on a special table which could be moved horizontally and vertically with micron-level precision. The beam dimensions were $5 \mathrm{~mm}$ wide (along the strips) and $0.5 \mathrm{~mm}$ high (transversely to the strips). During dynamic mode operation, the detector scanned the beam by moving in the transverse direction.

In Fig. 3 beam profile measurements, made in dynamic mode, are depicted. The distance between the two positions of the detector for these measurements is $400 \mu \mathrm{m}$. As one can see, the X-ray beam profiles are not symmetric and slightly different. This is explained by a beam intensity reduction, which has been confirmed by the data of the ionization chamber.

\section{Conclusions and outlook}

In summary, one may conclude that the developed technology to manufacture the micro-strip metal detector allows to realize non-destructive measurements of the intensity distribution of an X-ray beam, as well as to determine the beam profile with an accuracy as good as
$20 \mu \mathrm{m}$ in vacuum. The developed MMD also proved to be a reliable new tool for the charged particle radiation monitoring in a wide range of applications. We have performed studies demonstrating a good performance of the MMD in the focal plane of a mass-spectrometer. The further developments of the MMD will include:

- Improving the position resolution (to sub-micron level) by applying electron lithography for the strip pattern production,

- Applying microchip charge integrators and scalers,

- Developing different modifications of the MMD for particular applications.

\section{Acknowledgments}

We appreciate the essential help provided by A. Ehnes and $\mathrm{T}$. Kracht during the MMD tests at HASYLAB. These studies have been performed within the Agreement between DESY and KINR and with financial support from the DESY site.

\section{References}

[1] N.M. Tkatch, V.A. Kiva, Scientific Papers of the Institute for Nuclear Research, No. 2(4) (2001) 72.

[2] E.J. Sternglass, Phys. Rev. 108 (1957) 1.

[3] H. Rothard, et al., Springer Tracts in Modern Physics, Springer, Berlin, 1992, p. 97.

[4] O. Fedorovich et al., Plasma technologies for manufacturing of metal micro-strip detectors of ionizing radiation, 11th International Conference on Plasma Physics and Controlled Nuclear Fusion, 11-16 September 2006, Abstracts, Alushta 2006, p. 172.

[5] N. van Bakel, et al., The Beetle reference manual, Version 1.0, LHCb Note 2001-046, 2001.

[6] V. Pugatch, et al., Micro-strip metal foil detectors for the beam profile monitoring, Proc. DIPAC 2005, Lyon (France), 2005, p. 18. 